\title{
Calculating the Sun's Position for Calibration of Radar Antennas
}

\author{
from R. S. Blicq
}

ReCENTLy I was asked to assist the engineers in my company (a Canadian electronics firm) devise a method for using radiation from the Sun to check the alignment of heavy ground radar sets to within very fine tolerances. The degree of accuracy would have to be better than five minutes of arc in azimuth for search antennas, and three minutes of arc in elevation angle for height finding antennas. Solar radiation in the microwave region of the radio frequency spectrum would be used as a reference. The antenna reflector would be rotated in azimuth and elevation until maximum solar radiation was reflected into the waveguide horn and thence to the radar receiver. The moment peak detector current was obtained, the antenna would be locked in position and the exact G.M.T. noted. Normal astro-navigation calculation methods and tables would then be used to calculate the Sun's true azimuth and elevation angle, which would be compared with that measured by the antenna to determine its accuracy.

Since none of the engineers had previous navigation experience, they had run into difficulties when they tried to devise a method for calculating the Sun's position to within the requisite accuracy limits.

2. Difficulties and conditions. The first difficulty was the fact that most antenna calibrations would be performed by field technicians having no knowledge of astro-navigation tables and calculation methods. They would be at extremely remote radar stations across Canada and thus would not have an opportunity to ask questions if our instructions proved ambiguous. Consequently we decided we would have to develop an almost foolproof system of simple calculation forms accompanied by extremely explicit instructions.

This, however, proved difficult. Since the navigation tables could be entered only with whole degrees of L.H.A., latitude and declination, it would be necessary to interpolate for the additional minutes of arc in each case. It was these additional calculations that complicated the calculation forms beyond our expectations and considerably lengthened the step-by-step procedures that were envisaged.

We were aware that a single solar radiation measurement would be insufficiently accurate to determine antenna calibration; in fact, a minimum of ten actual measurements would have to be made and compared with the Sun's calculated position to obtain a satisfactory average error. To obviate the necessity for making a separate lengthy calculation for each, it was decided to make a series of calculations spaced at ten-minute intervals and to plot the results as a graph. The calculated angles could then be interpolated for the exact time of each measurement. Even then, several ten-minute calculations would be necessary since solar radiation measurements could not be taken in rapid succession: the manipulation of large antennas ( 30 to $50 \mathrm{ft}$. across) by hand is a relatively slow process.

On the other hand, since the antennas could be rotated vertically through only a limited angle of elevation, there would be a physical limit to the amount of 
time that could be expended on any one series of measurements. The time of day during which the Sun would be at a usable altitude would be relatively short; this is particularly true of search antennas, which can be rotated in elevation through only a very small angle above the horizon. Most of the year, approximately 35 minutes would be available twice daily for Sun azimuth measurements, and $1 \frac{1}{2}$ hours twice a day for elevation angle measurements. There would also be a brief period in mid-winter when at certain northern locations the Sun would be at a sufficiently low altitude most of the day.

Two other factors applicable only to height-finding radar sets also would have to be taken into consideration: refraction and weather. A correction for refraction could be obtained from pre-computed tables and applied to either the observed or calculated elevation angle. (In this context the term 'elevation angle', rather than 'height' or 'altitude', is used to denote the angle of the Sun above the horizon, since in height-finding radars the words 'height' and 'altitude' are used to denote target height above ground level.) In the case of weather, we would be unable to correct for deflection caused by the approach of a frontal system because the magnitude of the error could never be determined; therefore we would have to stipulate that relatively stable atmospheric conditions must always prevail in the direction of measurement.

Except in the case of a few $3-\mathrm{D}$ radars (those that measure azimuth and elevation angle simultaneously from one antenna system) only either elevation angle or azimuth is determined by any one radar set. Consequently two completely different calculation methods would be required: one for calibrating height finder radars, the other for calibrating search radars.

3. Elevation angle calculations. As in normal astro-navigation applications, elevation angle calculations involved the use of the Air Almanac and H.O. 249 (A.P. 3270 ) Sight Reduction Tables for Air Navigation, with which navigators are familiar, and a correction for refraction. A simple form was designed and gradually increased in scope as each problem was encountered. Throughout its birth pangs it was accompanied by an instruction sheet couched in terms that we considered could be followed by electronic technicians unfamiliar with astro-navigation terminology.

The form and detailed instructions were given to a representative crosssection of engineers and technicians so that difficulties and misunderstandings could be resolved as they arose. We were surprised how easily the uninitiated could be confused by apparently straightforward statements; the words 'height' and 'altitude', for instance, had to be changed to 'elevation angle' very early in the project. Whenever one of our guinea-pigs asked a question, or if there was the slightest element of doubt as to meaning, the pertinent area of the form or the text was amplified to ensure clarity. Gradually all ambiguities were resolved and the final form produced.

Items $A$ to $G$, at the top of the form, are mainly preliminary and provide basic information, such as L.H.A., declination and latitude, used in completing the balance of the form.

Because H.O. 249 can be entered using only whole degrees of L.H.A., latitude and declination, interpolation for the additional minutes of arc must be performed in each case. There are two entering arguments for latitude, each the whole degree below or above the actual site latitude. Similarly, there are two entering arguments for declination, each the whole degree below or above the Sun's actual declination; these head the LO DEC and HI DEC columns for both 
entering arguments of latitude. Finally, there are two entering arguments of L.H.A., each the whole degree above or below the actual L.H.A., which are entered against HI L.H.As and LO L.H.A. respectively on the left-hand side of the sheet. This arrangement permits a logical progression from H.O. 249 to the printed form.

The technician is instructed to turn to the LO L.A.T. pages of H.O. 249 and select the correct declination page. He then enters with HI L.H.A. and extracts the elevation angles $(\mathrm{HC})$ from LO DEC and HI DEC columns, which are adjacent to each other. He then re-enters with LO L.H.A. (just one line up or down), and again extracts the $\mathrm{Hc}$ for both declinations. All this information is entered into the LO LAT column of the form. The procedure is then repeated using the same entering arguments, but this time for the HI LAT column of the form.

Interpolation for L.H.A. is performed by multiplying the difference between the elevation angles for HI L.H.A. and LO L.H.A. by the odd minutes of L.H.A. divided by 60 . This is calculated for all four DEC columns and entered opposite DIFF FOR L.H.A. in each case. Because it is comparatively difficult to assess whether the difference should be subtracted from or added to the lower elevation angle entry, a note is printed on the form: 'if $(q)$ is greater than $(p)$, the sign is -ve'.

Interpolations for declination and latitude are performed in a similar manner; the results are entered against DIFF FOR DEC and DIFF FOR LAT respectively. Again, manipulation of the sign is resolved by notes. This results in the FINAL INTERPOLATED ELEVATION ANGLE.

A correction for refraction is obtained from a special graph (not from the Air Almanac) and is added to the calculated elevation angle. Thus all corrections are made to the calculated figure, the observed elevation angle remaining intact.

4. Azimuth Calculations. The azimuths provided by H.O. 249 proved insufficiently accurate for search radar calibration, consequently recourse had to be made to H.O. 260 Azimuths of the Sun. This volume was first printed in 1934 and has remained virtually unchanged since that time.

H.O. 260 requires three entering arguments: latitude and declination to the nearest whole degree, and apparent time to the nearest ten minutes. The front half of the volume contains latitude pages annotated 'declination same name as latitude'; the second half, pages annotated 'declination contrary name to latitude'. As in the H.O. 249 , declination columns from $0^{\circ}$ to $23^{\circ}$ head the top of each page. Apparent time is listed on both sides of every page, 'A.M' appearing on the left and 'P.M.' on the right. Azimuth to the nearest minute of arc is read from the body of the table.

A second form and an accompanying instruction sheet were prepared, tried out by the company's technical staff, amended and printed. In the form, items $A$ to $F$ are routine and provide the basic information for later calculations. Item L (declination) is completed at the same time as item D, when the G.H.A. is obtained from the Air Almanac. Latitude and longitude are determined to within ten seconds of arc to ensure optimum accuracy.

G.M.T. rounded-off to the nearest ten minutes is selected for simplicity when entering the Air Almanac and determining L.H.A.; the L.H.A. is then converted to apparent time, in hours, minutes and seconds. However, apparent time rounded-off to the nearest ten minutes has to be used as the entering argument for the H.O. 260. The G.M.T. for this rounded-off apparent time is determined by subtracting the original apparent time from the rounded-off 
apparent time, and applying the result with the same sign to the original G.M.T. These steps are performed at items $G$ to $K$ on the form. The rounded-off apparent time and equivalent G.M.T. are entered at the head and foot respectively of Calculation No. I ; ten minutes is added to each to provide the times for Calculation No. 2.

The entering arguments for declination, each the whole degree below or above the Sun's actual declination, head the LO DEC and HI DEC columns. Similarly, the entering arguments of latitude, each the whole degree above or below the actual site latitude, are entered against $\mathrm{HI}$ LAT and LO LAT respectively on the left-hand side of the sheet. This arrangement permits a logical progression through the H.O. 260. The technician is instructed to select the HI LAT page of the H.O. 260 (depending on whether the declination is 'same name as' or 'contrary name to' the latitude), enter with the apparent time of Calculation No. I and extract the azimuths from the LO DEC and HI DEC columns. He then re-enters with the apparent time of Calculation No. 2 and extracts the azimuths from the same declination columns. This whole procedure is repeated with the same entering arguments, but this time using the LO LAT page of the H.O. 260.

Interpolation for latitude and declination is performed in the same manner as that for the elevation angle calculations. The results are entered against DifF FOR LAT and DIFF FOR DEC respectively, the former always being additive; manipulation of the sign for the difference in declination is simplified by a note.

Since the highest declination column in the H.O. 260 is $23^{\circ}$, difficulty can exist twice a year when the Sun's declination is greater than $23^{\circ}$. Under these conditions the technician is instructed to use $22^{\circ}$ for LO DEC and $23^{\circ}$ for HI DEC, and to proceed normally. The only additional step he must take is to add 60 minutes to the odd minutes of declination when computing DIFF FOR DEC; this is stated on the form in a note.

5. Practical application. Before releasing the forms and instructions to the field technicians, I accompanied a small team of engineers to an operational radar site and tested the system. Navigators would have found this sight-taking phase particularly interesting: imagine taking manually-operated sextant observations to a very high degree of accuracy with a sextant that is 55 feet in height and weighs over six tons! To add to our problems, the receiver, and thus the detector current meter, was on the floor below the antenna, which necessitated using a telephone system for communication. And all this was done in a radome which, by the end of a summer's day, recorded a temperature of 1 i $8^{\circ} \mathrm{F}$.

This practical evaluation proved entirely successful except in one area: the width of the radiation band was found to be rather narrow and thus initially it was very difficult to locate the Sun electrically. Since the antenna was housed in a fibreglass radome having a polyester resin coating, neither was it possible to align the antenna visually.

We had decided not to make any calculations prior to taking solar radiation measurements since, if for any reason the measurements could not be performed at the planned time, to do so would have necessitated a lot of unnecessary work. Thus we had to devise a simple form on which approximate azimuth and elevation angle could be calculated quickly at 20 -minute intervals. This was done and, once the antenna had been set to the approximate location, it proved relatively simple to 'search' vertically and horizontally until an increase in receiver detector current showed that solar radiation was being received. The instructions were amended to include the use of this additional form. 
The system has now been in use for some time. More recently, the lengthy calculations required by our system have been replaced by daily sheets of azimuth and elevation angles prepared by electronic computer for each site. However, a hand-computed system still has its uses: for calibrating mobile radar sets, and for new installations before the precomputed sheets are available.

\title{
The Gestation and Birth of my Nautical Tables
}

\author{
from S. M. Burton
}

(An Address to the Association of Navigation Schools, Grimsby, 18 May 1962)

THE imaginary question I propose to answer in this address is-how does it come about that a person with such a very ordinary sort of intellect, and certainly with no particular flair for mathematics, should be the author, or nominal author, of such an imposing publication as a volume of nautical tables?

To build up the background to the story briefly let me explain that in my training ship days I received the impression that a ship's position was found by mathematical calculations. True, we knew that sextants and chronometers were necessary instruments in the scheme of things; but they only seemed to supply certain ingredients to the calculations that ultimately produced one of the components, latitude or longitude. It was when I was consigned to a stretch of service on the North Pacific in 1927 that, for something to do, I decided to try and improve my limited understanding of astronomical navigation. In the volume of Raper's Tables which I had always used there was an appendix by the Naval Instructor William Hall which explained what was then called by the Navy 'The New Navigation', and gave four-figure cosine-versine tables for the work. It was in reading, and re-reading, and thinking about Hall's marvellously lucid explanation of the St. Hilaire process that the light suddenly burst upon me. It became revealed to me that zenith distance is the same as geographical distance; and that when you take a sight you measure your actual distance from the body in homely nautical miles. What was still more interesting was-that this must always have been the case, both before, as well as after, the invention of the chronometer.

Now, this may sound a bit trite. But let it be remembered that Sumner discovered his line in 1837 , and it was not until some seventy-odd years later that St. Hilaire realized what it was that Sumner had discovered, and suggested an obvious new angle of approach to the whole subject. St. Hilaire did not invent or suggest any new mathematical process, as far as I know. Even I, in my training ship days had been taught how to solve the 'third side direct' as the problem was then called in spherical trigonometry.

Well, this discovery quite went to my head, and I rushed around the ship metaphorically shouting Eureka and telling my navigational colleagues about the 